\title{
Machine-Learning Based Stacked Ensemble Model for Accurate Analysis of Molecular Dynamics Simulations
}

\author{
Samrendra K. Singh ${ }^{1}$, Karteek K. Bejagam ${ }^{2}$, Yaxin $\mathrm{An}^{2}$, Sanket A. Deshmukh ${ }^{2 *}$
}

1. CNH Industrial, Burr Ridge, Illinois 60527, United States

2. Department of Chemical Engineering, Virginia Tech, Blacksburg, Virginia 24061, United States

*Email: sanketad@vt.edu

\section{S1. Machine Learning (ML) Models:}

Random/Regression Forest: The Random forest (RF) is an ensemble of several decision trees (" $n$ " trees). ${ }^{1}$ Each decision tree in the RF, based on its application, acts as a regressor (in contact angle SEM) or as a classifier (in hydrogen bond SEM). The original training dataset of RF is bootstrapped to create " $n$ " number of training set, one for each tree. ${ }^{2}$ A bootstrapped dataset is created by resampling from the original dataset and can have repeated data points one or more times. A decision tree is built by splitting the training data at several levels (branches) such that each end node (leaf) has similar data points (features) as shown in Fig. S1. ${ }^{3}$ The homogeneity of the data in a node is achieved by recursively splitting the data based on the Gini value. ${ }^{4}$ A leaf from a classifier decision tree corresponds to one output result (a discrete value), whereas a regression model is fit into the dataset of a node to predict a continuous value (real number). ${ }^{5}$ In addition to making predictions, a decision tree can also provide information about the importance of each input features affecting the output. The most important feature forms the root of the tree followed by other important features forming the branches. The branch splitting terminates at nodes with uniform data points are called leaves. ${ }^{6}$ 


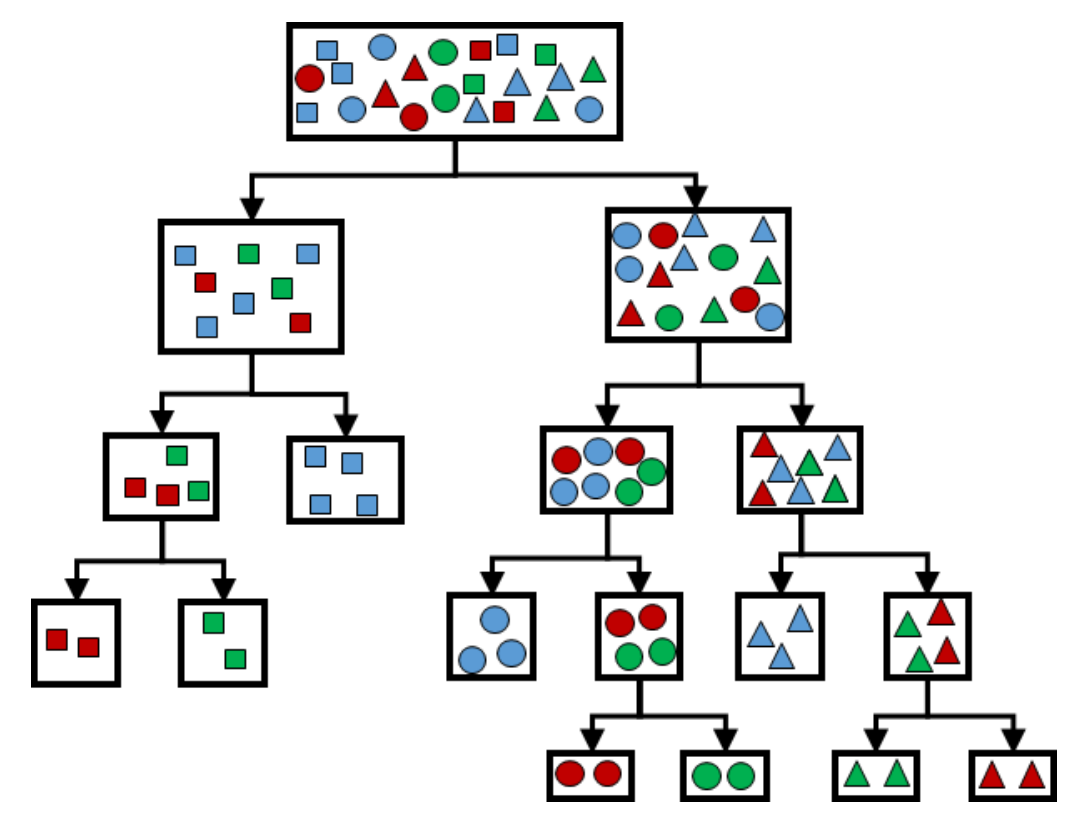

Figure S1: Schematic of a decision Tree.
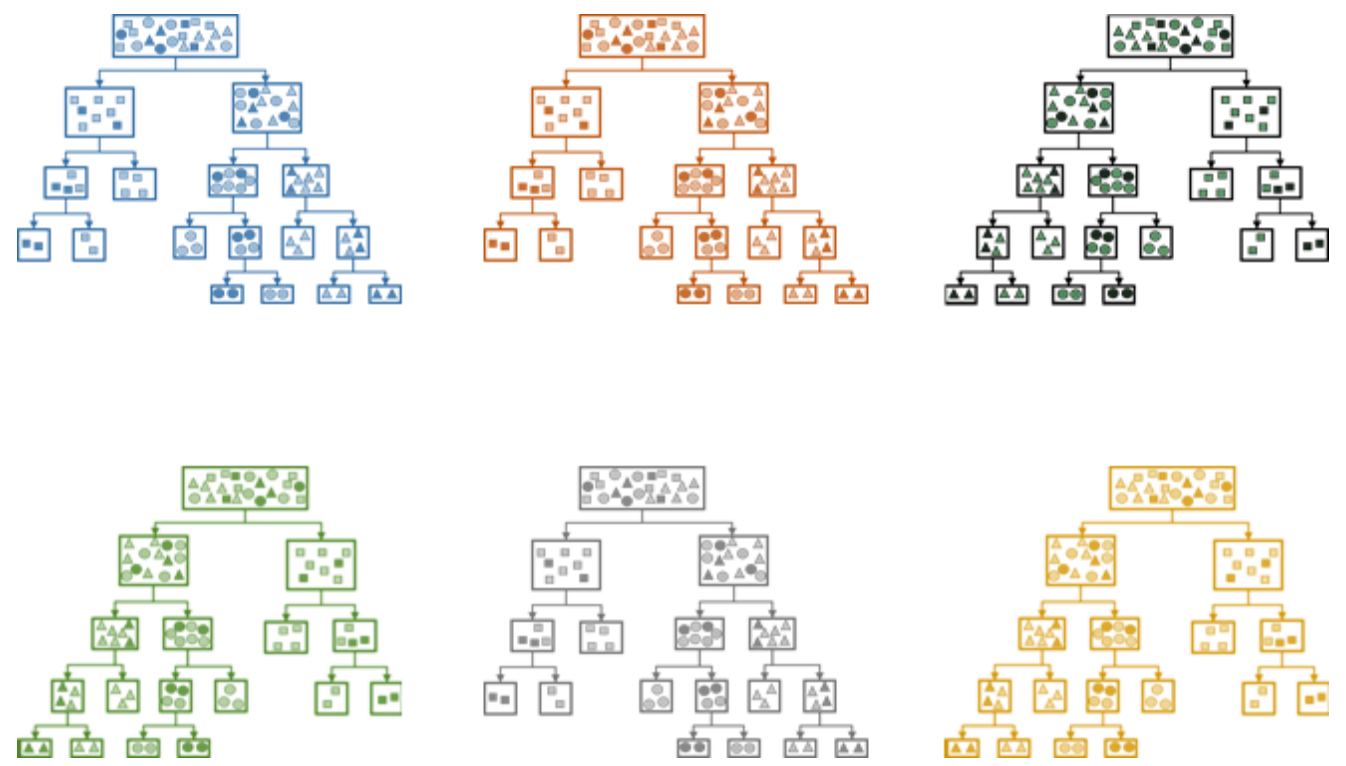

Figure S2: Schematic of a random forest made of several decision trees.

The RF model is an ensemble approach by using several decision trees together to build a predictive model, as shown in Fig. S2. The final prediction of a RF model is based on either voting for the predicted value by member trees and picking the majority vote in case of classifier or averaged value, in case of a regressor. ${ }^{7}$ RF models are simple in construction and yet are 
robust against overfitting. They make reliable predictions with sufficiently large number of trees and training data size. Due to its simplistic architecture, it's one of the fastest ML methods. We used the standard library available in sklearn kit for python. ${ }^{8}$ The details of architectures for all four RF models developed in the present study are shown in Table S1.

Artificial Neural Network (ANN): In recent years, artificial Neural Network (ANN) have been commonly utilized in the field of science and engineering to build predictive models. ${ }^{9-13}$ As shown in Fig. S3, an ANN model is consist of several layers, where each layer contains several nodes. A node is formed of a summation function and an activation function. The nodes in any given layer is connected to every node in the preceding and succeeding layers. The input for a given node comes from the nodes of preceding layer, which is then multiplied with a weighting factor corresponding to each input node in the preceding layer. The weighted input signal is then added and passed through an activation function. We used the ReLu as activation function for the ANN models developed in the present study. ${ }^{14,15}$ The output from the activation function of a node is transmitted to the nodes in the next layer. The weightage factors of each node is modified using an optimization method, with objective to minimize the square mean error between the actual and predicted dataset. For a classifier a tanh or step activation function is typically used to convert the real number into a discrete value. The details of the two ANN models developed in the present study are listed in Table $\mathbf{S 1}$.

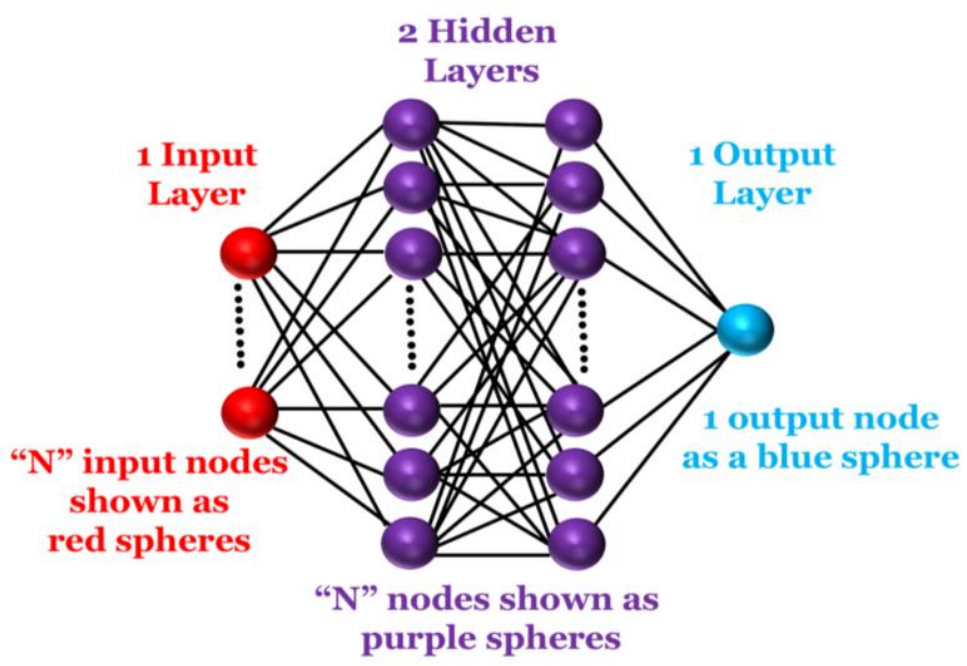

Figure S3: Schematic of the ANN model. 
Support Vector Regression (SVR): Support vector regression (SVR) is an extension of the more popular Support Vector Machine (SVM) ML model. ${ }^{16,17}$ An SVM is typically used as a classifier, whereas the SVR is a regressor variance of the SVM. ${ }^{18}$ Similar to SVM, the SVR works based on creating a hyperplane in $n$-dimensional feature space such that the plane is able to divide the input dataset into smaller homogeneous fragments. ${ }^{19,20}$ A hyperplane is constructed by using a linear combination of input features (Eq. S1). The coefficients describing a hyperplane $\left(a_{1}, a_{2}, \ldots, a_{n}\right.$ and $\left.b\right)$ are optimized such as to maximize the margin/gap ( $\delta$ ) between the hyperplane and the data-points.

$$
a_{1} \cdot x_{1}+a_{2} \cdot x_{2}+\ldots \ldots+a_{n} \cdot x_{n}+b=0 \ldots \ldots \ldots . . \text { Equation } \mathbf{S 1}
$$

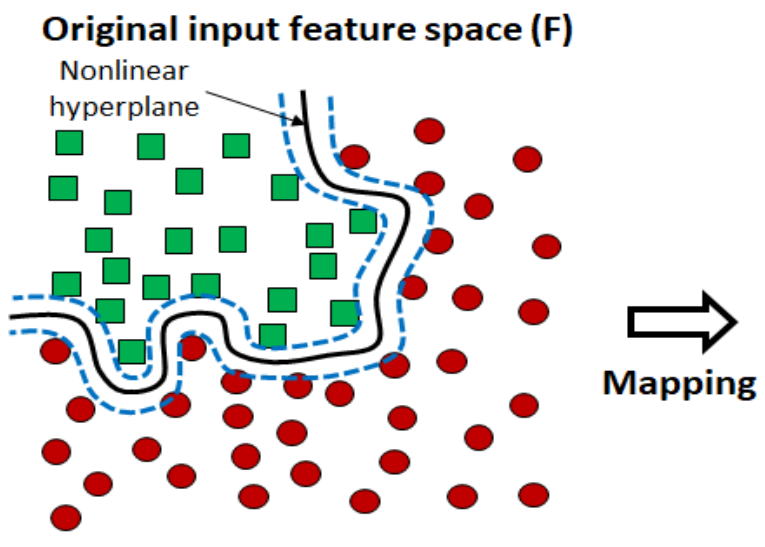

(a)

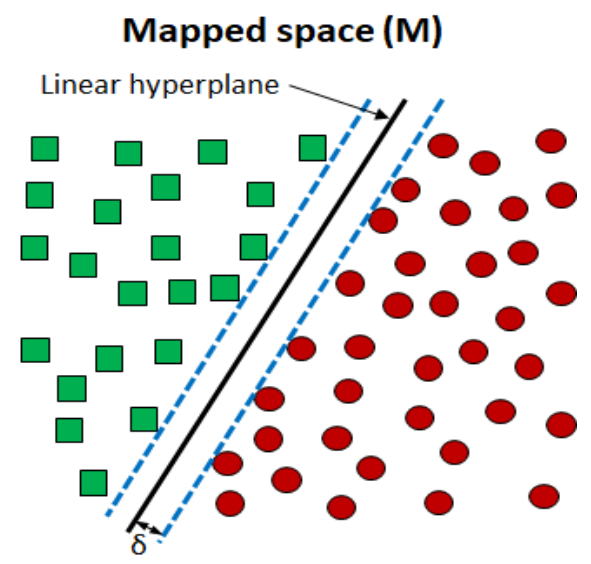

(b)

Figure S4: The schematic showing mapping of data points from original feature space (a) to the mapped feature space (b), where the points can be separated using linear hyperplane dividing the two classes. The black line represents an example hyperplane in 2D feature space and the blue dotted line represent the margin between the hyperplane and the data points at the separation boundary.

The structure risk minimization (SRM) segregates the non-linear dataset into separate subsets with the help of the kernel-trick method. The kernel-trick method performs a nonlinear mapping of the low-dimensional input feature dataset into a higher dimensional feature space, where the data is linear and highly separable. The hyperplane is then created in the higher dimensional feature space, mapped from the original input feature space (Fig. S4). There are several kernel functions e.g. gaussian Radial Basis Function (RBF), linear, polynomial and 
sigmoid to name a few. ${ }^{21}$ We used the most popular RBF for our model (Eq. S2), where $k\left(x_{i}, x_{j}\right)$ is the kernel function between two feature points $x_{i}$ and $x_{j}$ and $\sigma^{2}$ is the variance.

$$
k\left(x_{i}, x_{j}\right)=e^{\left(-\frac{\left|x_{i}-x_{j}\right|^{2}}{2 \sigma^{2}}\right)} \ldots \ldots \ldots \ldots \text { Equation S2 }
$$

The model hyperparameters developed and used in the present study are listed in Table S1.

Kernel Ridge Regression (KRR): Kernel Ridge Regression (KRR) is one of the kernel based regression method, which maps the features from lower dimensional space to a higher dimensional space using kernel-trick. ${ }^{22}$ KRR is very similar to SVM, specially the non-linear version. The main difference between the SVM and KRR is that they minimize different objective functions. The KRR method adds a regularized penalty term based on the norm of the hyperparameters, to the objective function. ${ }^{23}$ Unlike the SVM, the KRR doesn't give extra weightage to the points close to the dividing boundaries (hyperplane), but rather depends upon all points in the given data set. One drawback of KRR is poor scaling with increasing size of the training dataset. The hyperparameters developed in the present study are listed in Table S1.

k-Nearest Neighbors $(\boldsymbol{k}-N \boldsymbol{N})$ : k-Nearest Neighbors (k-NN) uses a simple approach of comparing a given input feature vector with the training feature vectors and finds $\mathrm{k}$ closest matching points (Fig. S5) ${ }^{24-26}$ A point refers to a feature vector of size $n$ in n-dimensional space. The proximity of a given input point (feature vector) with the training points (training features) is calculated based on a euclidean distance function (Eq. S3) ${ }^{27-29}$ A classifier type of k-NN gives an output in the form of membership of the majority of the $\mathrm{k}$ points in the neighborhood. On the other hand, a regression type application's output is the average property associated with $\mathrm{k}$ points in the neighbourhood. For our models, we used $\mathrm{k}=2$, which means everytime we entered an input feature vector to our k-NN model, it searched for 2 points (training features) closest to it based on the Eq. S3, where $x$ and $y$ are two features. Then returned the average value of the contact angle corresponding to those two points as an output. k-NN models are highly localized and can perform very well with large data set spread uniformly across the feature space. The model parameters for the two models employed in this study are listed in Table S1. 


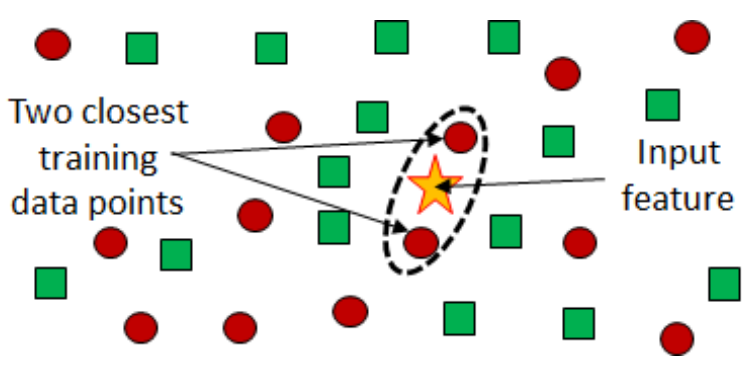

Figure S5: A schematic of working principal of the k-NN algorithm. The algorithm looks for $\mathrm{k}$ nearest features from the training set to make the prediction.

Table S1: Model design/hyperparameters used for the individual ML models of the SEM

\begin{tabular}{|c|c|c|}
\hline & $\begin{array}{c}\text { Training data } \\
\text { set id }\end{array}$ & Architecture/Hyper-parameters \\
\hline RF \#1 & 1 & Number of trees $=200$, max dept $=25$ \\
\hline RF \#2 & 2 & Number of trees $=100$, max dept $=30$ \\
\hline ANN \#1 & 3 & \# hidden layers $=5,10-24-12-6-6-3-1$, activation func=ReLU \\
\hline ANN \#2 & 4 & \# hidden layers=4, 10-20-10-5-3-1, activation func $=$ ReLU \\
\hline SVM \#1 & 5 & kernel=rbf, tol=1e-09, C=10000, eps $=0.1$ \\
\hline SVM \#2 & 6 & kernel=rbf, tol=2e-04, C=10000, eps=0.1 \\
\hline kNN \#1 & 7 & n_neighbors $=3$ \\
\hline kNN \#2 & 8 & n_neighbors $=2$ \\
\hline KRR \#1 & 9 & kernel=poly, alpha=1e-9,coef0=0.1, degree=3 \\
\hline KRR \#2 & 10 & kernel=poly, alpha=1e-9,coef0=0.1, degree $=3$ \\
\hline
\end{tabular}




\begin{tabular}{|c|c|c|}
\hline RF \#3 & 11 & Number of trees $=100$, max dept $=10$ \\
\hline RF \#4 & 12 & Number of trees $=100$, max dept $=10$ \\
\hline
\end{tabular}

Stacking of the ML models: There is an inherent error associated with every ML model emanating from the sources like noise, variance, and bias. By grouping several ML models, one can reduce the variance greatly and increase the stability of the overall model prediction. The stacking ensemble model (SEM) combines several relatively weak models to create a robust and accurate model.

\section{S2. Generation of a $200 \AA$ diameter water droplet:}

A simulation cell of $500 \AA$ x $500 \AA$ x $500 \AA$ was created and a water droplet with $200 \AA$ diameter with $\sim 150,000 \mathrm{SPC} / \mathrm{Fw}$ molecules was created. The LAMMPS simulation package was used to perform the molecular dynamics (MD) simulations. The droplet was equilibrated for $\sim 1$ ns with a timestep of $1 \mathrm{fs}$ at $300 \mathrm{~K}$. The periodic boundary conditions were applied in all three directions, and simulations were performed in NVT ensemble. A cut-off of $12 \AA$ was applied for non-bonded interactions. Simulation trajectory was stored every 1 ps, and last 2 frames from the 500 ps simulation trajectory were used to train the ML models in the SEM. To ensure that we consider all the atoms in droplet, we remove atoms that are evaporated (beyond $5 \AA$ of the edge of the droplet).

\section{S3. Evaluating the performance of SEM for Contact Angle Predictions:}

The performance of any regression model is typically quantified with Root Mean Square Error $(R M S E)$, Mean Absolute Percentage Error $(M A P E)$ and correlation coefficient $\left(R^{2}\right)$ as shown in Eq. S4 to S7. The actual value is represented by y and the predicted quantity by $x$ for $\mathrm{n}$ number of predictions.

$$
\begin{aligned}
& \text { RMSE }=\sqrt{\frac{1}{n} \sum_{i=1}^{n}\left(y_{i}-x_{i}\right)^{2}} \\
& \text { MAPE }=\frac{1}{n} \sum_{i=1}^{n} \frac{\left|y_{i}-x_{i}\right|}{y_{i}} \times 100 \\
& R^{2}=\left[\frac{C v(x, y)}{\sqrt{\operatorname{Cv(x,x)Cv(y,y)}}}\right]^{2}
\end{aligned}
$$$$
\text { Equation S4 }
$$$$
\text { Equation S5 }
$$$$
\text { Equation S6 }
$$ 


$$
C v(x, y)=\frac{\sum_{i=I}^{N}\left(x_{i}-\bar{x}\right)\left(y_{i}-\bar{y}\right)}{n-1}
$$

Table S2: The performance metrics of all ML models in the SEM for contact angle.

\begin{tabular}{|c|c|c|c|c|c|c|}
\hline & \multicolumn{3}{|c|}{ Predictions Stats } & \multicolumn{3}{c|}{ Uncertainty Quantification of the residuals } \\
\hline & RMSE & $\begin{array}{c}\text { MAPE } \\
(\%)\end{array}$ & $\mathbf{R}^{2}$ & Mean error & 95\% min_limit & 95\% max_limit \\
\hline RF \# 1 & 2.316 & 2.202 & 0.9946 & 1.60 & 1.508 & 1.692 \\
\hline RF \# 2 & 2.205 & 2.078 & 0.9951 & 1.54 & 1.447 & 1.633 \\
\hline ANN \# 1 & 2.766 & 2.916 & 0.9922 & 2.04 & 1.935 & 2.144 \\
\hline ANN \#2 & 2.080 & 2.139 & 0.9956 & 1.50 & 1.421 & 1.579 \\
\hline SVR \# 1 & 1.212 & 1.073 & 0.9985 & 0.74 & 0.685 & 0.795 \\
\hline SVR \# 2 & 1.268 & 1.016 & 0.9984 & 0.74 & 0.681 & 0.799 \\
\hline k-NN \# 1 & 2.857 & 2.515 & 0.9917 & 2.00 & 1.887 & 2.113 \\
\hline k-NN \# 2 & 2.889 & 2.418 & 0.9915 & 2.06 & 1.943 & 2.177 \\
\hline KRR \# 1 & 1.339 & 1.311 & 0.9982 & 0.92 & 0.866 & 0.974 \\
\hline KRR \# 2 & 1.379 & 1.372 & 0.9981 & 0.96 & 0.905 & 1.015 \\
\hline RF \# 3 & 0.942 & 0.849 & 0.9991 & 0.62 & 0.579 & 0.661 \\
\hline RF \# 4 & 0.986 & 0.8762 & 0.999 & 0.62 & 0.578 & 0.662 \\
\hline Final & 0.912 & 0.8024 & 0.9992 & 0.58 & 0.541 & 0.619 \\
\hline
\end{tabular}


(a)

\section{0}

\section{5}

2.0
1.5

1.5

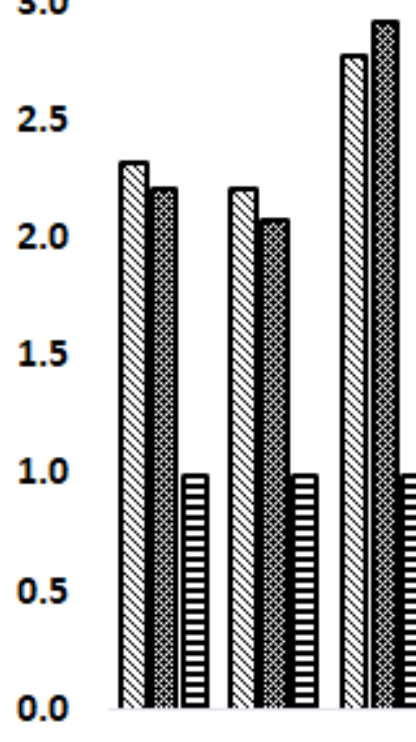

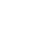
s*

$2.5 \quad$ (b)

2.0

2.0

1.5 1.0

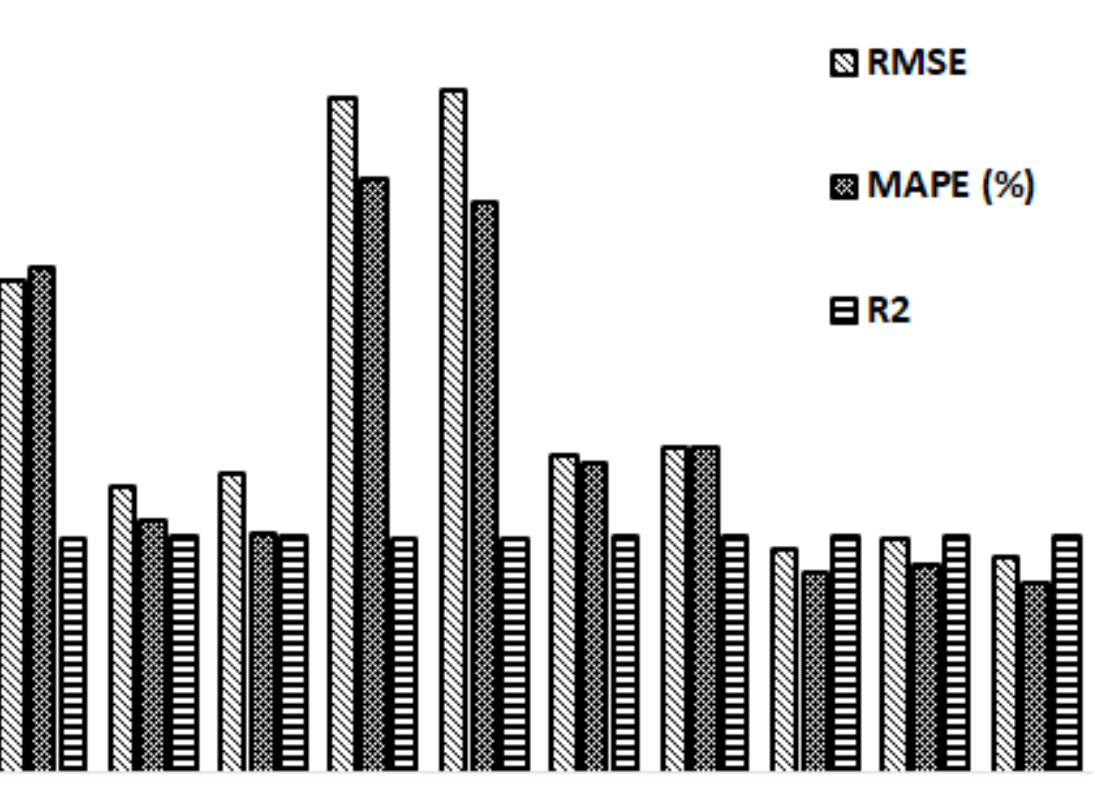


Figure S6: Performance metrics of the SEM. (a) The prediction performance indicators of the individual models and final prediction of the SEM. (b) The average percentage error calculated from uncertainty quantification of residuals using bootstrapping technique for each ML model of the SEM. The $95 \%$ confidence interval is shown by vertical lines on top of the bars.

\section{S4. MD Simulations of a Water Droplet on a 2-D Substrate:}

All-atom MD simulations of a water droplet on the two-dimensional graphene sheet were performed using LAMMPS package. ${ }^{30}$ Graphene sheet was represented using REBO potentials and water potentials were adopted from TIP3P/Fs. ${ }^{31-33}$ The flexible graphene sheet is infinite in $\mathrm{X}$ and $\mathrm{Y}$ directions through periodic boundary conditions. The dimensions of graphene sheet are $159.56 \AA$ x $159.14 \AA$ in $\mathrm{X}$ and Y directions, respectively. The initial configuration consists of the 2000-molecule water droplet placed at a distance of $\sim 7 \AA$ from the graphene sheet. The simulations were performed at $300 \mathrm{~K}$ in the NVT ensemble for $1.2 \mathrm{~ns}$. The time-step for integrating the equations of motions was 1 fs. The contact angle for a given simulation was determined by analyzing the last 400 ps employing the recently developed method based on the circular fit. ${ }^{34}$ In our previous work, we have developed the cross-interactions represented by 12-6 Lennard-Jones potentials between the graphene, represented using REBO potentials and five commonly used water models employing particle swarm optimization. ${ }^{34}$ The developed crossinteractions for water modelled using TIP3P/Fs and graphene sheet were $0.32679 \mathrm{~kJ} / \mathrm{mol}$ and $3.19 \AA$ for epsilon (interaction strength between carbon and oxygen atom) and sigma (the distance at which the interaction becomes zero), respectively. Note, the interactions between the hydrogen and carbon atoms were not considered. These potentials have been successfully demonstrated to reproduce the contact angle of $97^{\circ}$ for the macroscopic water droplet consistent with the experimental and theoretical studies. ${ }^{35,36}$ During the development of cross-interactions, we have shown that the strength of interaction is inversely proportional to the contact angle of water. ${ }^{34}$ Therefore, to validate the ML model across wide range of contact angle values, we have conducted $93 \mathrm{MD}$ simulations by varying the epsilon values from 0.048 to $0.6753 \mathrm{~kJ} / \mathrm{mol}$ so that the contact angle of water varies from $\sim 140^{\circ}$ (hydrophobic) to $\sim 30^{\circ}$ (hydrophilic). Note, 0.32679 $\mathrm{kJ} / \mathrm{mol}$ was able to reproduce the contact angle of $97^{\circ}$ for the macroscopic water droplet. 
(a)

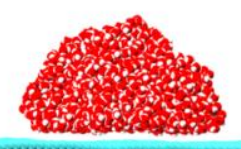

(b)

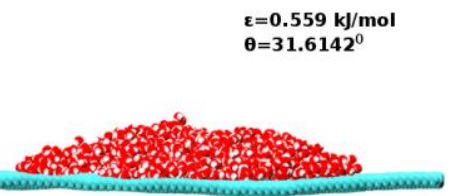

(c)

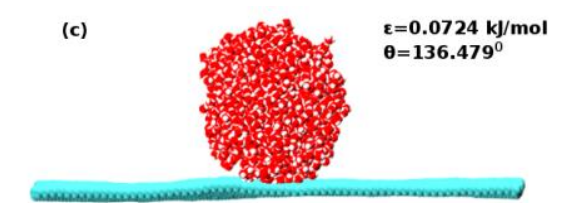

Figure S7: (a) Initial configuration of water droplet on the graphene sheet. Equilibrated structure of water droplet on the graphene sheet with different interaction strength between oxygen atom and graphene carbon. (b) and (c) depict the representative snapshots of hydrophilic and hydrophobic surface, respectively.

\section{S5. Sensitivity Analysis of SEM Regressor:}
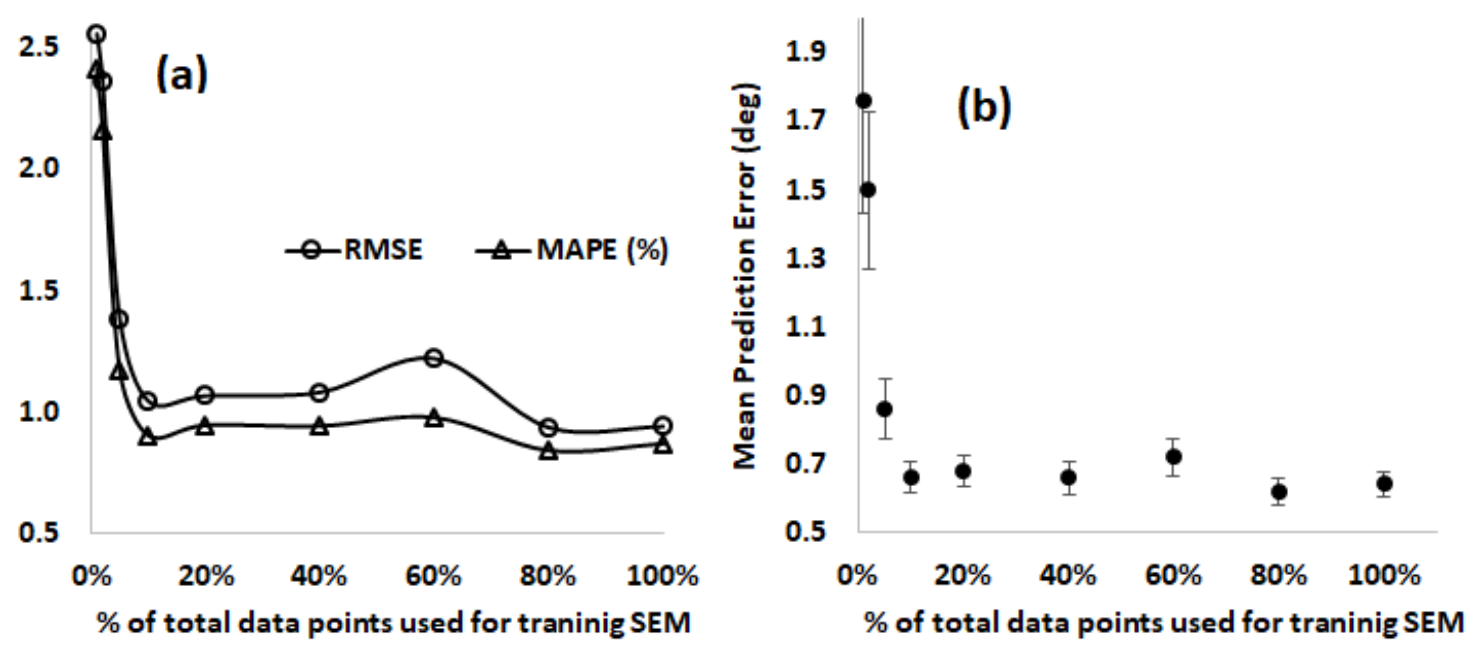

Figure S8: Sensitivity analysis of SEM for contact angle prediction based on training data size. (a) RMSE and MAPE, (b) Mean prediction error from bootstrapping uncertainty analysis. The bars on the points in (b) represent $95 \%$ confidence intervals.

Table S3. The performance metrics of the contact angle SEM with different size of training data set.

\begin{tabular}{|c|c|c|c|c|c|c|}
\hline Data (\%) & RMSE & $\begin{array}{c}\text { MAPE } \\
(\%)\end{array}$ & $\mathbf{R}^{2}$ & Mean error & $\begin{array}{c}\text { 95\% } \\
\text { min_limit }\end{array}$ & $\begin{array}{c}\text { 95\% } \\
\text { max_limit }\end{array}$ \\
\hline $1 \%$ & 2.560 & 2.416 & 0.992 & 1.760 & 1.433 & 2.087 \\
\hline $2 \%$ & 2.362 & 2.158 & 0.995 & 1.500 & 1.271 & 1.729 \\
\hline $5 \%$ & 1.382 & 1.168 & 0.998 & 0.860 & 0.770 & 0.950 \\
\hline
\end{tabular}




\begin{tabular}{|c|c|c|c|c|c|c|}
\hline $10 \%$ & 1.044 & 0.903 & 0.999 & 0.660 & 0.616 & 0.704 \\
\hline $20 \%$ & 1.066 & 0.945 & 0.999 & 0.680 & 0.633 & 0.727 \\
\hline $40 \%$ & 1.081 & 0.942 & 0.999 & 0.660 & 0.612 & 0.708 \\
\hline $60 \%$ & 1.220 & 0.977 & 0.999 & 0.720 & 0.666 & 0.774 \\
\hline $80 \%$ & 0.936 & 0.841 & 0.999 & 0.620 & 0.581 & 0.659 \\
\hline $100 \%$ & 0.940 & 0.870 & 0.999 & 0.640 & 0.602 & 0.678 \\
\hline
\end{tabular}

S6. Evaluating the performance of SEM for H-Bond predictions

Table S4: Bootstrapping error obtained for the individual ML models in hydrogen bond SEM.

\begin{tabular}{|c|c|c|c|}
\hline & \multicolumn{3}{|c|}{ Bootstrapping Error } \\
\hline & Mean error (\%) & $95 \%$ min_limit & $95 \%$ max_limit \\
\hline RF \# 1 & 1.27 & 1.01 & 1.53 \\
\hline RF \# 2 & 1.32 & 1.07 & 1.57 \\
\hline ANN \# 1 & 1.70 & 1.41 & 1.99 \\
\hline ANN \#2 & 1.44 & 1.17 & 1.71 \\
\hline SVR \# 1 & 1.19 & 0.95 & 1.43 \\
\hline SVR \# 2 & 1.07 & 0.83 & 2.07 \\
\hline k-NN \# 1 & 1.78 & 1.49 & \\
\hline
\end{tabular}




\begin{tabular}{|c|c|c|c|}
\hline k-NN \# 2 & 1.70 & 1.42 & 1.98 \\
\hline KRR \# 1 & 1.07 & 0.82 & 1.32 \\
\hline KRR \# 2 & 1.07 & 0.82 & 1.32 \\
\hline RF \# 3 & 0.91 & 0.69 & 1.13 \\
\hline RF \# 4 & 0.90 & 0.69 & 1.11 \\
\hline Final & 0.89 & 0.66 & 1.12 \\
\hline
\end{tabular}

Several statistical measures as shown in Eq. S8 - S13, were used to evaluate the performance of the classifier models of SEM. ${ }^{37-39}$ The performances of the individual ML models along with the final prediction of the SEM is shown in the Fig. S9, and S10.

$$
\begin{aligned}
& \text { Sensitivity }(S e)=\frac{T P}{T P+F N} \\
& \text { Specificity }(S p)=\frac{T N}{T N+F P} \\
& \text { Precision }(P r)=\frac{T P}{T P+F P} \\
& \text { Error }(\operatorname{Er})=\frac{F P+F N}{T P+T N+F P+F N} \\
& \text { Accuracy }(A c)=\frac{T P+T N}{T P+T N+F P+F N}
\end{aligned}
$$

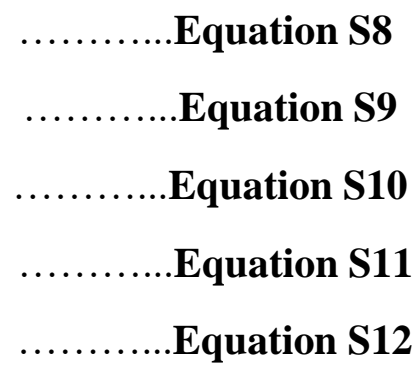

Balanced CLassification Rate $(B C R)=\frac{\text { Sensitivity }+ \text { Specificity }}{2}$ Equation S13

Table S5: Definitions of TP, FP, TN, FN

\begin{tabular}{|c|c|c|c|}
\hline Symbol & Meaning & Actual & Predicted \\
\hline TP & True Positive & 1 & 1 \\
\hline FP & False Positive & 0 & 1 \\
\hline
\end{tabular}




\begin{tabular}{|c|c|c|c|}
\hline TN & True Negative & 0 & 0 \\
\hline FN & False Negative & 1 & 0 \\
\hline
\end{tabular}

(a)

Sensitivity

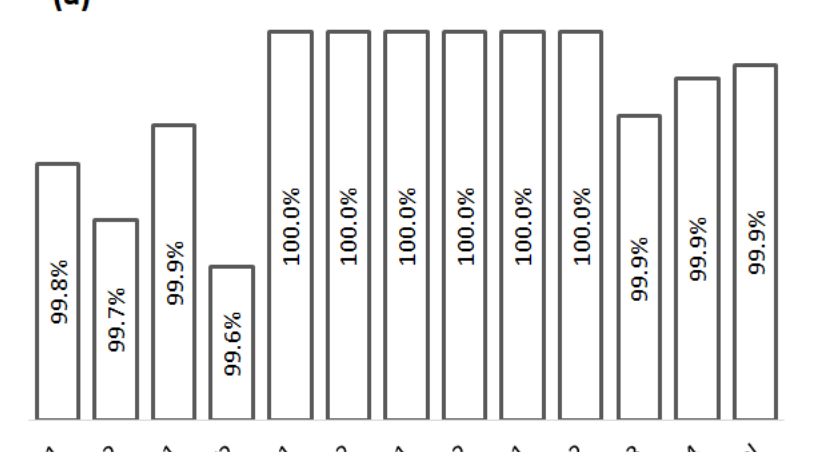

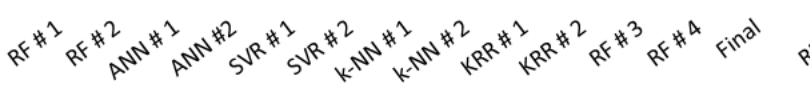

(c)

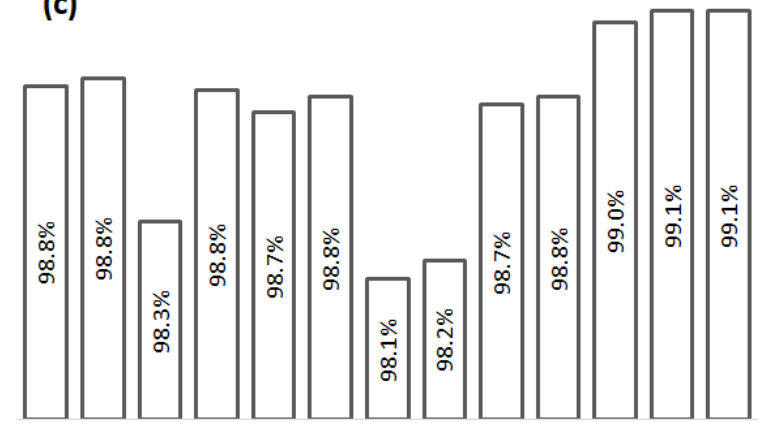

(b)

(b)

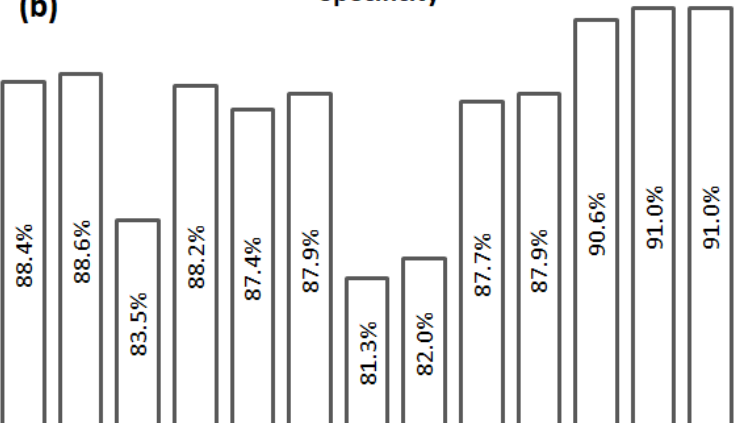

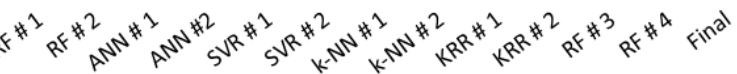

(d)

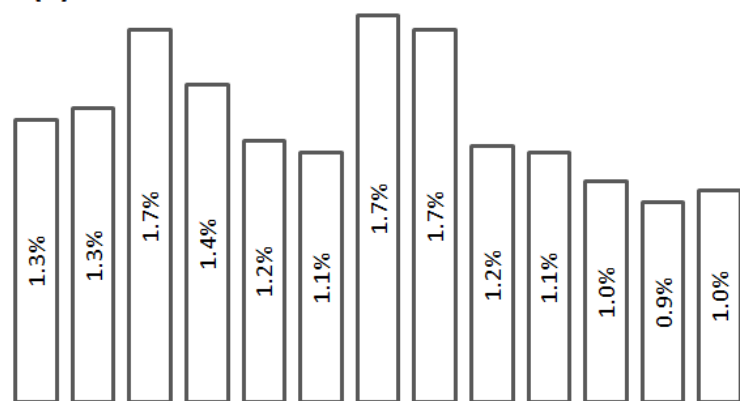

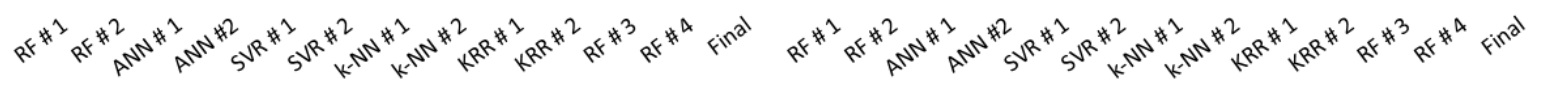

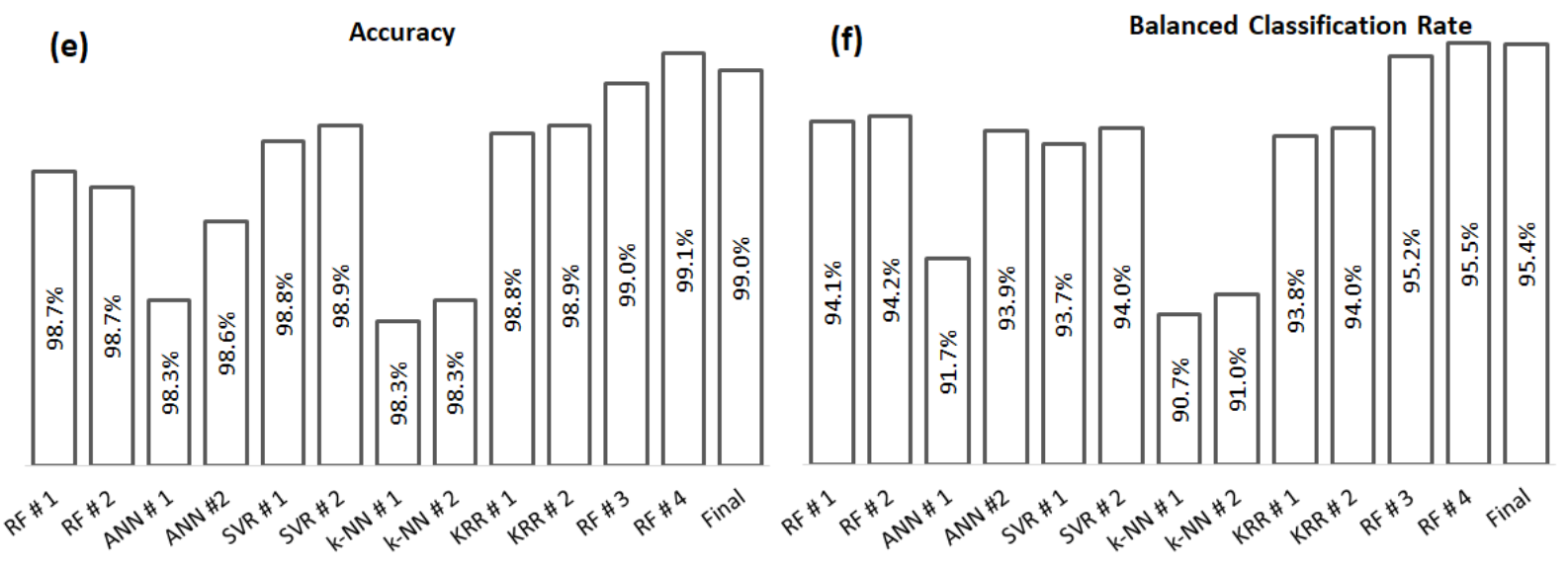


Figure S9: Statistical measures for performance of the ML models and the SEM (a) Sensitivity (b) Specificity (c) Precision (d) Error (e) Accuracy, and (f) Balanced classification rate

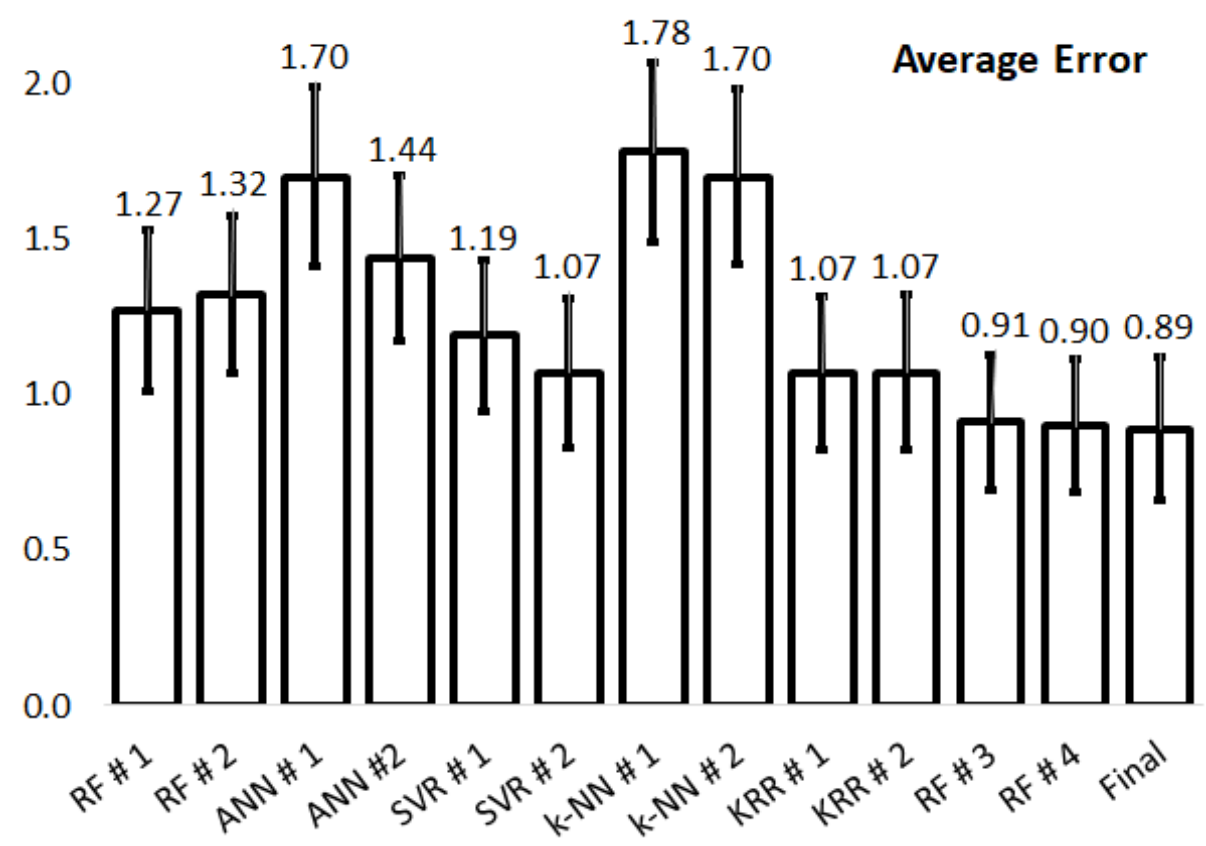

Figure S10: Average percentage error associated with the individual ML models, and final output of the SEM model for predicting hydrogen bonds calculated by bootstrapping technique. The $95 \%$ uncertainty range is represented by the lines at the top of the bars.

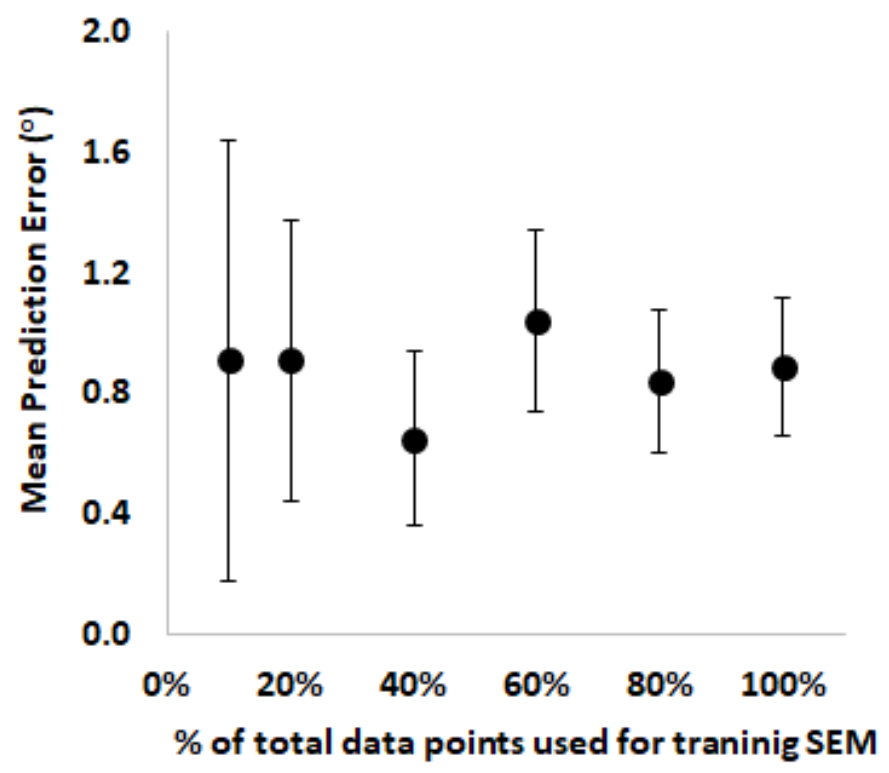

Figure S11: Sensitivity analysis of the SEM for prediction of hydrogen bond. The $95 \%$ confidence interval is represented by the vertical lines at each point. 
Table S6: Statistical measures and uncertainty quantifications of the SEM for hydrogen bond, trained with different sizes of training data

\begin{tabular}{|l|l|l|l|l|l|l|l|l|l|}
\hline \multicolumn{7}{|l|}{ Classifier Statistical Measures } \\
\hline $\begin{array}{l}\text { Data } \\
(\%)\end{array}$ & Se & Sp & Pr & Er & Ac & BCR & $\begin{array}{l}\text { Mean } \\
\text { error }\end{array}$ & $\begin{array}{l}\text { 95\% } \\
\text { min } \\
\text { limit }\end{array}$ & $\begin{array}{l}\text { 95\% max } \\
\text { limit }\end{array}$ \\
\hline $10 \%$ & $99.9 \%$ & $89.6 \%$ & $99.0 \%$ & $1.1 \%$ & $98.9 \%$ & $94.7 \%$ & 0.910 & 0.182 & 1.638 \\
\hline $20 \%$ & $99.9 \%$ & $91.3 \%$ & $99.1 \%$ & $0.9 \%$ & $99.1 \%$ & $95.6 \%$ & 0.910 & 0.447 & 1.373 \\
\hline $40 \%$ & 100.0 & $92.0 \%$ & $99.3 \%$ & $0.7 \%$ & $99.3 \%$ & $96.0 \%$ & 0.650 & 0.360 & 0.940 \\
\hline $60 \%$ & $99.9 \%$ & $88.7 \%$ & $98.9 \%$ & $1.1 \%$ & $98.9 \%$ & $94.3 \%$ & 1.040 & 0.741 & 1.339 \\
\hline $80 \%$ & $99.9 \%$ & $91.6 \%$ & $99.1 \%$ & $0.9 \%$ & $99.1 \%$ & $95.8 \%$ & 0.840 & 0.605 & 1.075 \\
\hline $100 \%$ & $99.9 \%$ & $91.0 \%$ & $99.1 \%$ & $1.0 \%$ & $99.0 \%$ & $95.4 \%$ & 0.890 & 0.662 & 1.118 \\
\hline
\end{tabular}

\section{References:}

(1) Breiman, L. Random Forests. Mach. Learn. 2001, 45 (1), 5-32.

(2) Hirsch, R. M.; Archfield, S. A.; De Cicco, L. A. A Bootstrap Method for Estimating Uncertainty of Water Quality Trends. Environmental Modelling \& Software 2015, 73, 148166.

(3) Yu, Z.; Haghighat, F.; Fung, B. C. M.; Yoshino, H. A Decision Tree Method for Building Energy Demand Modeling. Energy Build. 2010, 42 (10), 1637-1646.

(4) Farid, D.; Zhang, L.; Rahman, C. M.; Hossain, M. A.; Strachan, R. Hybrid Decision Tree and Naïve Bayes Classifiers for Multi-Class Classification Tasks. Expert Syst. Appl. 2014, 41 (4, Part 2), 1937-1946.

(5) Silins, V.; Julien, F.; Brasher, C.; Nivoche, Y.; Mantz, J.; Dahmani, S. Predictive Factors of PACU Stay after Herniorraphy in Infant: A Classification and Regression Tree Analysis. Paediatr. Anaesth. 2012, 22 (3), 230-238.

(6) Quinlan, J. R. Simplifying Decision Trees. Int. J. Man. Mach. Stud. 1987, 27 (3), 221-234.

(7) Breiman, L. Classification and Regression Trees; Routledge, 2017.

(8) Pedregosa, F.; Varoquaux, G.; Gramfort, A.; Michel, V.; Thirion, B.; Grisel, O.; Blondel, 
M.; Prettenhofer, P.; Weiss, R.; Dubourg, V.; et al. Scikit-Learn: Machine Learning in Python. J. Mach. Learn. Res. 2011, 12 (Oct), 2825-2830.

(9) Singh, S.; Abbassi, H. 1D/3D Transient HVAC Thermal Modeling of an off-Highway Machinery Cabin Using CFD-ANN Hybrid Method. Appl. Therm. Eng. 2018, 135, 406-417.

(10) Reich, Y.; Barai, S. V. Evaluating Machine Learning Models for Engineering Problems. Artificial Intelligence in Engineering 1999, 13 (3), 257-272.

(11) Arhami, M.; Kamali, N.; Rajabi, M. M. Predicting Hourly Air Pollutant Levels Using Artificial Neural Networks Coupled with Uncertainty Analysis by Monte Carlo Simulations. Environ. Sci. Pollut. Res. Int. 2013, 20 (7), 4777-4789.

(12) Devika, P. D.; Achenie, L. E. K. On the Use of Quasi-Newton-Based Training of a Feedforward Neural Network for Time Series Forecasting. J. Intell. Fuzzy Syst. 1995, 3 (4), 287-294.

(13) Bejagam, K. K.; Singh, S.; An, Y.; Deshmukh, S. A. Machine-Learned Coarse-Grained Models. J. Phys. Chem. Lett. 2018, 9 (16), 4667-4672.

(14) Eckle, K.; Schmidt-Hieber, J. A Comparison of Deep Networks with ReLU Activation Function and Linear Spline-Type Methods. Neural Netw. 2019, 110, 232-242.

(15) Yarotsky, D. Error Bounds for Approximations with Deep ReLU Networks. Neural Netw. 2017, 94, 103-114.

(16) Basak, D.; Pal, S.; Patranabis, D. C. Support Vector Regression. Neural Information Processing-Letters and Reviews 2007, 11 (10), 203-224.

(17) Schölkopf, B.; Smola, A. J.; Managing Director of the Max Planck Institute for Biological Cybernetics in Tubingen Germany Profe Bernhard Scholkopf; Bach, F. Learning with Kernels: Support Vector Machines, Regularization, Optimization, and Beyond; MIT Press, 2002.

(18) Gunn, S. R. Support Vector Machines for Classification and Regression. ISIS technical report 1998.

(19) Widodo, A.; Yang, B.-S. Support Vector Machine in Machine Condition Monitoring and Fault Diagnosis. Mech. Syst. Signal Process. 2007, 21 (6), 2560-2574.

(20) Boser, B. E.; Guyon, I. M.; Vapnik, V. N. A Training Algorithm for Optimal Margin Classifiers. Proceedings of the fifth annual workshop on Computational learning theory COLT '92. 1992. https://doi.org/10.1145/130385.130401.

(21) Géron, A. Hands-On Machine Learning with Scikit-Learn and TensorFlow: Concepts, Tools, and Techniques to Build Intelligent Systems; “O'Reilly Media, Inc.," 2017.

(22) An, S.; Liu, W.; Venkatesh, S. Face Recognition Using Kernel Ridge Regression. In 2007 IEEE Conference on Computer Vision and Pattern Recognition; 2007; pp 1-7.

(23) Ogutu, J. O.; Schulz-Streeck, T.; Piepho, H.-P. Genomic Selection Using Regularized Linear Regression Models: Ridge Regression, Lasso, Elastic Net and Their Extensions. BMC Proc. 2012, 6 Suppl 2, S10.

(24) Fukunage, K.; Narendra, P. M. A Branch and Bound Algorithm for Computing KNearest Neighbors. IEEE Trans. Comput. 1975, No. 7, 750-753.

(25) Keller, J. M.; Gray, M. R.; Givens, J. A. A Fuzzy K-Nearest Neighbor Algorithm. IEEE Trans. Syst. Man Cybern. 1985, SMC-15 (4), 580-585.

(26) Horton, P.; Nakai, K. Better Prediction of Protein Cellular Localization Sites with the It K Nearest Neighbors Classifier. Ismb 1997.

(27) Seidl, T.; Kriegel, H.-P. Optimal Multi-Step K-Nearest Neighbor Search. In Proceedings of the 1998 ACM SIGMOD International Conference on Management of Data; SIGMOD 
'98; ACM: New York, NY, USA, 1998; pp 154-165.

(28) Qian, G.; Sural, S.; Gu, Y.; Pramanik, S. Similarity Between Euclidean and Cosine Angle Distance for Nearest Neighbor Queries. In Proceedings of the 2004 ACM Symposium on Applied Computing; SAC '04; ACM: New York, NY, USA, 2004; pp 1232-1237.

(29) Shahabi, C.; Kolahdouzan, M. R.; Sharifzadeh, M. A Road Network Embedding Technique for K-Nearest Neighbor Search in Moving Object Databases. Geoinformatica 2003, 7 (3), 255-273.

(30) Plimpton, S. Fast Parallel Algorithms for Short-Range Molecular Dynamics. J. Comput. Phys. 1995, 117 (1), 1-19.

(31) Brenner, D. W.; Shenderova, O. A.; Harrison, J. A.; Stuart, S. J.; Ni, B.; Sinnott, S. B. A Second-Generation Reactive Empirical Bond Order (REBO) Potential Energy Expression for Hydrocarbons. J. Phys. Condens. Matter 2002, 14 (4), 783.

(32) Wu, Y.; Tepper, H. L.; Voth, G. A. Flexible Simple Point-Charge Water Model with Improved Liquid-State Properties. J. Chem. Phys. 2006, 124 (2), 024503.

(33) Schmitt, U. W.; Voth, G. A. The Computer Simulation of Proton Transport in Water. $J$. Chem. Phys. 1999, 111 (20), 9361-9381.

(34) Bejagam, K. K.; Singh, S.; Deshmukh, S. A. Development of Non-Bonded Interaction Parameters between Graphene and Water Using Particle Swarm Optimization. J. Comput. Chem. 2017. https://doi.org/10.1002/jcc.25141.

(35) Shih, C.-J.; Wang, Q. H.; Lin, S.; Park, K.-C.; Jin, Z.; Strano, M. S.; Blankschtein, D. Breakdown in the Wetting Transparency of Graphene. Phys. Rev. Lett. 2012, 109 (17), 176101.

(36) Taherian, F.; Marcon, V.; van der Vegt, N. F. A.; Leroy, F. What Is the Contact Angle of Water on Graphene? Langmuir 2013, 29 (5), 1457-1465.

(37) Kovalishyn, V.; Aires-de-Sousa, J.; Ventura, C. QSAR Modeling of Antitubercular Activity of Diverse Organic Compounds. Chemometrics Intellig. Lab. Syst. 2011.

(38) Gaba, S.; Jamal, S.; Open Source Drug Discovery Consortium; Scaria, V. Cheminformatics Models for Inhibitors of Schistosoma Mansoni Thioredoxin Glutathione Reductase. ScientificWorldJournal 2014, 2014, 957107.

(39) Weis, D. C.; Visco, D. P., Jr; Faulon, J.-L. Data Mining PubChem Using a Support Vector Machine with the Signature Molecular Descriptor: Classification of Factor XIa Inhibitors. J. Mol. Graph. Model. 2008, 27 (4), 466-475. 